\title{
FOLDING WORK LOGISTICS IN SERIAL PRODUCTION
}

\author{
Gunars Verdins ${ }^{1}$, Dace Rotgalve-Birziete ${ }^{1}$, Martins Berzins ${ }^{2}$ \\ ${ }^{1}$ Latvia University of Life Sciences and Technologies, Latvia; ${ }^{2}$ SIA "HansaMatrix Ventspils “, Latvia \\ gunars.verdins@1lu.lv, dace.rotgalve@1lu.1v,mr.berzin@gmail.com
}

\begin{abstract}
Mechanical engineering companies with serial production, including agricultural, communal and specialized machinery production, handle a relatively large number of parts on bending presses. The large variety of parts in terms of dimensions, weight and shapes require specific equipment of different capacities and sizes. Typically, companies buy presses that currently provide all possible bending work. In the best case, it would be most advantageous to use an appropriate power and gauge press for each bended part, the price of which would also be lower. Companies require the knowledge, where it is more advantageous to make the relevant parts - in their own company with the equipment they contain, or in cooperation with a company with more appropriate equipment and lower costs. In order to make such calculations, a company or any other researcher should have accurate information about the cost of bending work. In the thesis, a detailed methodology for standardization of bending work has been elaborated, folding work for series of small, bulky and complex parts for timing the work. The obtained data on the time capacity of the measures are needed to produce the parts. Based on the results obtained, the cost of bending work for small, medium and large series is calculated. The work cost analysis is performed. The output parameters for organization of the part folding work are determined, depending on the number of parts in the series, the geometrical parameters of the component, the number of folds, the complexity of the component shape, the effort required, the number of punches and matrices to be used, the weight of the parts, the component material and other factors. There is a methodology for organizing part folding works in a mechanical engineering company for small, medium and large series production.
\end{abstract}

Keywords: folding work, bended parts, time recording, rationing, cost, prime cost.

\section{Introduction}

One of the most effective technological solutions for producing volumetric parts in agriculture, communal vehicle manufacturing and specialized machine production companies with serial production is laser cutting flat pattern parts and folding them with bending presses. The resulting part quality and production time is greatly affected by organization of work and rationing. The required time for folding and processing can be analysed with several methods. Inspecting and measuring folding work with a stopwatch, it is possible to determine and estimate the required bending time, process core elements, maintenance and other time values required to perform current folding work $[1 ; 2]$. A normator is required for this specific methodology, although his presence may be disturbing the operators' work and his ability to concentrate on the given task. Another method is to film video, where the presence of the normator and stopwatch is replaced with a video camera, therefore, the filmed material with inspected work can be repeated several times or even played in slow motion to capture even the finest details and to obtain precise time frames for each individual performed action during folding work [3;4]. The regression calculation method is based on pre-measured patterns of work operations. Based on these data, a regression calculation is performed, after which the formula for calculating the planned time is created. The resulting formula is implemented in a time calculation system, which calculates the time required to produce other parts $[5 ; 6]$. In cases where the production time for a component has to be determined within a short period of time, an indicative time calculation method is used. Such method is observed by practice of experienced specialists and is approximate time estimation, which has no quantitative basis, but qualitative [7]. In serial production conditions, the most effective method for inspecting bending work is the video timing method. Compared to the timekeeping method, where the estimator keeps records in the vicinity of the employee, the video surveillance method does not require the presence of an observer at the workplace. The captured video can be repeated several times and also in slow motion, thus obtaining more accurate data [8;9].

The aim of the thesis is the peculiarities of bending works in companies with serial production and to propose a strategy for improvement of bending work organization. In order to achieve the goal, it is necessary to perform experimental research on the process of folding works by filming the process of manufacturing various parts with video surveillance, to process data with parameters, to select individual parts representing the groups, to compare technologies of selected parts, to perform data 
analysis, based on the results obtained, to work out the standardization method of bending works and operative management strategy in companies with small, medium and large series production.

\section{Materials and methods}

During inspection of folding work, a digital video camera with 12-megapixel photo resolution and video recording with $5 \mathrm{x}$ optical zoom function is used. Manufacturing processes are observed of producing parts with different size and complexity level, which are made of S235J2 steel with the sheet thickness from 2 to $6 \mathrm{~mm}$. Bending work on bulky parts is done on the 4-axis bending press TruBend 5170S (maximum bending length of 3.2 meters, maximum compression force of $1700 \mathrm{kN}$ ) and the 6-axis bending press TruBend 5085 (maximum bending length 2.2 meters, max. compressive force of $850 \mathrm{kN}$ ). The experiment is performed during the day working shift in serial production circumstances. Flat pattern parts are delivered to the bending station and placed in a specially designated area, which does not interfere with the movement of the operator through the experimental area and is relatively close to the bending press. Based on the previously obtained results [8], folding work time capacity calculation formulas have been developed. The whole process of folding works is divided into separate stages, parameters are chosen that characterize separate groups of parts. The total folding time $T_{t o t}$ adds up to the operating time $T_{o p}$, instrument tying time $T_{i n}$ and the time for recreation $T_{r e}$ :

$$
T_{t o t}=T_{o p}+T_{i n}+T_{r e}
$$

The operational time is summed up from the basic time directly for the folding time $T_{\text {bas }}$, the base time-related auxiliary time $T_{a u x}$ and the time used to control the component $T_{c o n}$ :

$$
T_{o p}=T_{b a s}+T_{a u x}+T_{c o n},
$$

The tool tying time $T_{\text {in }}$ ads up to the tool set up time $T_{\text {com }}$, tool setting time $T_{\text {set }}$ and the tool feed time $T_{\text {feed }}$ :

$$
T_{i n}=T_{\text {com }}+T_{\text {set }}+T_{\text {feed }},
$$

The total time of component folding adds the time of recreation time $T_{r e}$, drawing analysis time $T_{\text {ras }}$, program input time $T_{p r}$, and wasted time $T_{n e l}$.

The base time for $T_{b a s}$ is calculated from:

$$
T_{\text {bas }}=\left(n \cdot T_{\text {one }}+T_{\text {com }}\right) \cdot T_{\text {con }} \cdot K,
$$

where $n$-number of folds;

$T_{\text {one }}$ - time of creation of one fold, min;

$K-$ coefficient.

The value of the coefficient $K$ is determined by the condition that one fold is made, the length of the fold line is $100 \mathrm{~mm}$, the bending angle is $90^{\circ}$, the radius of curvature of the auxiliary tool is $5 \mathrm{~mm}$. The coefficient values increase with increasing the length of the bending line and range from 0.8 to 1.27 [8].

The time of creating one bend is determined by experiments, calculating the arithmetic mean of many measurements. Summarizing the expressions, we get the expression of the total folding time $T_{\text {sum }}$ :

$$
T_{\text {sum }}=T_{\text {bas }}+T_{\text {aux }}+T_{\text {con }}+T_{\text {com }}+T_{\text {set }}+T_{\text {feed }}+T_{\text {re }}+T_{\text {ras }}+T_{p r}+T_{\text {nel }},
$$

This expression can be used, if the individual values are known. The countable value varies depending on the group of parts. It is assumed that the bending lines may not be in the same plane. All parts are divided into 3 groups: Small S, Bulky B and Complex C. The representative of each group's component analysis results in a representative that describes the most accurate details. Small-sized bended parts are with a linear dimension from 16 to $500 \mathrm{~mm}$, with a number of bending lines from 1 to 6. The category of large bended parts consists of linear dimensions from 500 to $1200 \mathrm{~mm}$, with a number of bending lines ranging from 1 to 6 . The category of sophisticated curved parts includes parts 
with linear dimensions ranging from $1200 \mathrm{~mm}$ to $2636 \mathrm{~mm}$, with a number of bending lines between 6 and 9. Parts are added to the complex category if: the component weight exceeds $25 \mathrm{~kg}$; the overall dimensions of the component exceed $1.20 \mathrm{~m}$; more than 5 folds provided; designed with 2 or more bending radius; folds are not in the same plane; contains tight output tolerances; the fold line is not parallel to the outer contour of the part. Particularly, parts with a weight of over $40 \mathrm{~kg}$ are specially marked, because in such cases, pneumatic vacuum suction grippers should be used, or two operators must perform folding simultaneously to produce such parts.

This expression can be used, if the individual values are known. The countable value varies depending on the group of parts. Bending lines may not be in the same plane. All parts are divided into 3 groups: Small- S, Big- B and Complex- C. The results of each group's component analysis are determined by a representative, which is the most representative of these parts. The category of smallgauge curved parts is with a linear dimension from 16 to $500 \mathrm{~mm}$, with a number of bending lines from 1 to 6. Big are divided into categories with linear dimensions of 500 to $1200 \mathrm{~mm}$, the number of bending lines being from 1 to 6 . The category of complex parts includes parts with linear dimensions ranging from $1200 \mathrm{~mm}$ to $2636 \mathrm{~mm}$, with a number of bending lines between 6 and 9 .

It is assumed that the drawing reading time $T_{\text {ras }}$ is a constant value of 15 seconds, which is the arithmetic mean of the experiment performed.

The Tool Shuttle tool feed rate is the factory-set size that cannot be changed in the control panel, and therefore the feed time of any machine drawer is 10 seconds. The time required to find the tool drawer for the task to be performed in the Tool Shuttle library is set in the experiment and fixed for 13 seconds.

The amount of time it takes to get the selected bending aids from the Tool Shuttle is 20 seconds.

The length of the folding line determines the minimum length of the active auxiliary tools needed for the bending press to perform the particular bending operation. The length of the active auxiliary tools is a numerical value that determines the number of units of auxiliary tools and the amount of weight relative to the total length of the auxiliary tool to be set.

The number of curved lines of folded parts affects the time of production of the part, significantly increasing the number of required actions associated with creation of each fold. Creating individual folding lines may include several additional steps to obtain the desired result, which increases the production time of the component.

The ancillary tool set-up and replacement time consists of a set of actions to be performed by press release to prepare and release tools before and after the task. Additional time for setting and replacing tools includes: manual adjustment of the angle of bending with the angle, linear dimension check, manual adjustment of the bending angle, manual correction of the bending program. The auxiliary tool set-up and replacement time is applied as a constant value of 1 minute.

Grouping of parts by complexity includes several factors: the weight of the part exceeds $25 \mathrm{~kg}$; the overall dimensions of the component exceed $1.20 \mathrm{~m}$; more than 5 folds provided; Designed for 2 or more bending radii; folds are not in the same plane; certain small tolerances; the fold line is not parallel to the outer contour of the part. Particularly, over $40 \mathrm{~kg}$ parts are marked because pneumatic vacuum suction cups have to be used in such cases, or two workers have to make such parts.

The auxiliary time related to the base time includes loading of the hydraulic press of the bending press, calibration of the tool jaw, setting of the optical safety sensor, opening and closing of the side doors, adjustment of the linear position of the auxiliary tools.

\section{Results and discussion}

The experimentally determined percentages of individual average relative values [8] for different group parts are summarized in Table 1 .

The total production time of the 10-piece batch of small parts is 9.77 minutes, of which the bending time is 2.85 minutes, basic time-inevitably tied time is 2.38 minutes; with tool setting after bending length -2.46 minutes, quality control time -0.32 minutes, drawing readout time -0.25 minutes, program load time -0.5 minutes. The length of one bend is $8.6 \mathrm{~s}$. 
Experimentally determined percentages for different group parts

Table 1

\begin{tabular}{|c|c|c|c|c|c|c|c|c|c|}
\hline Parametrs & $\boldsymbol{T}_{\text {bas }}$ & $\boldsymbol{T}_{\text {aux }}$ & $\boldsymbol{T}_{\text {con }}$ & $\boldsymbol{T}_{\text {com }}$ & $\boldsymbol{T}_{\text {set }}$ & $\boldsymbol{T}_{\text {feed }}$ & $\boldsymbol{T}_{\text {ras }}$ & $\boldsymbol{T}_{\text {pr }}$ & $\boldsymbol{T}_{\text {nel }}$ \\
\hline Small & 32.0 & 27.0 & 4.0 & 0 & 15.0 & 13.0 & 3.0 & 6.0 & 0 \\
\hline Big & 16.14 & 23.78 & 12.97 & 6.63 & 16.57 & 6.20 & 2.16 & 4.32 & 11.2 \\
\hline Complex & 18.33 & 22.78 & 10.84 & 2.69 & 11.69 & 2.86 & 0.63 & 1.26 & 28.92 \\
\hline
\end{tabular}

The total production time of big parts is 11.57 minutes, of which the bending time is 1.87 minutes, basic unavoidable time is 2.75 minutes, tool set-up time 0.77 minutes, tool setup and amount of feed time associated with tool wrapping length -2.63 minutes, quality control time -1.5 minutes, drawing readout time -0.25 minutes, program load time -0.5 minutes, and the wasted use time -1.3 minutes. The length of one bend is $9.3 \mathrm{~s}$.

The total production time of complicated parts is 39.65 minutes, of which the basic time of bending works is 7.27 minutes, basic unavoidable time is 9.03 minutes; amount of feed time associated with tool setting after bending line length -5.76 minutes, quality control time -4.3 minutes, drawing readout time -0.25 minutes, program load time -0.5 minutes, and the wasted use time -11.47 minutes. The length of one bend is $12.9 \mathrm{~s}$.

Comparing the results, it can be seen that in the process of making large and complicated parts, the amount of time spent on complicated parts is about $29 \%$ and for big parts about $11 \%$ of the total time.

The experimental data show that for the preparation of the bending process, including the program for inserting and drawing the drawing of small parts, 3.62 minutes are used, for big parts it is 5.44 minutes, and for complicated parts -19.03 minutes.

When classifying serial production for small series, medium and large series production, the calculations assume that the production of small batches of parts takes up to 4 hours. From the following conditions it can be determined that $90.55 \%$ of the time spent for small part production and control is used for the production of small parts, while $90.20 \%$ of the time is spent for manufacturing and controlling big parts, while $87.0 \%$, when the complicated parts are manufactured and controlled.

Provided that the price of the press $P$ and equipment $A(P+A)$ is 170 thousand EUR (5), electric motor power $7 \mathrm{~kW}$, electricity price $0.20 \mathrm{EUR} \cdot \mathrm{h}^{-1}$, operator hourly rate $8 \mathrm{EUR}$, the press with equipment must pay off over 5 years, we make one machine hour cost calculation (6). The cost of one machine hour COSh is determined by dividing the production cost COS with the working time of the press h.

Manufacturing costs per year are calculated from (6):

$$
C O S=\frac{(P+A)}{5}+E k s p l+E l+E n+T e l p,
$$

Operating and repair costs Ekspl are calculated by multiplying the price of the press and equipment by 0.05 , to get 8.500 EUR per year. The depreciation or interest rate of the machine En is calculated by assessing the initial value, utilization rate, time in years and the cost per hour [7]. $\mathrm{En}=170000 \cdot 0.07=11900$ EUR per year.

Electricity costs $E l$ are determined by multiplying the electric motor power $\mathrm{W}(\mathrm{kW})$ by the price of one kilowatt hour, the intensity of use $(0.20)$ and the duration of use (hours per year). $E l=7 \cdot 0.2 \cdot 0,20 \cdot 8 \cdot 1700=3808$ EUR a year. The cost of space Telp is calculated by multiplying the cost of renting one square meter of $\mathrm{m}^{2}\left(\mathrm{Cm}^{2}=4 \mathrm{EUR}\right)$, by the area occupied by the press $\left(\mathrm{Loc}=12 \mathrm{~m}^{2}\right)$ and equipment and by the length of the months $($ Tmont $=12)$. The calculation result in: Telp $=\mathrm{Cm}^{2} \cdot$ Loc $\cdot$ Tmont $=4 \cdot 12 \cdot 12=576$ EUR per year. By summing up the production costs, we get: COS $=34000+8500+3808+11904+576=58788$ EUR.

Working in one shift, the press working time $h P$ is determined by subtracting the time spent on holidays (12.8), personal needs and rest, which is $7 \%$ of $(2088-96=1992 \mathrm{~h}) 1992 \mathrm{~h}=139 \mathrm{~h}$. The result is that $h P=1853 \mathrm{~h}$. This is the case, if the machine is serviced and repaired outside the working hours. 
As a result, the cost of one machine hour is: $\operatorname{COSh}=58788 / 1853=31.73$ EUR per hour, or 0.53 EUR per minute.

Operator labour costs are summed up by the operator's salary, vacation and sickness expenses, administration and maintenance costs. The operator's salary is determined by multiplying the time worked by the hourly rate. The time worked is determined by deducting the time spent on leave and diseases, which is 30 days or 240 hours. The result is $1853-240=1613 \mathrm{~h}$. The operator receives the gross working time of $1613 \cdot 8=12904$ EUR. Additionally, holiday money and sickness money $(240 \cdot 7=1680$ EUR $)$ should be added. Administration costs $30 \%$ of the operator's gross salary are $12904 \cdot 0.30=3871$ EUR. As a result, we get the cost of work for PS is 18455 EUR. The cost of one working hour is $18455 / 1622=11.38$ EUR. Summing up machine hours and hourly costs, we get one hour of folding work, which is $31.73+11.38=43.11$ EUR per hour, or 0.72 EUR per minute. When calculating the minimum required press force $\mathrm{F}$, we assume that the arc is $6 \mathrm{~mm}$ thick at $90^{\circ}$ angles and the length of the folding line is max. The force required for folding parts is calculated by:

$$
F=\frac{1.6 \cdot b \cdot t^{2} \cdot R m}{L(N)},
$$

where $b$-width of the workpiece at the fold, $\mathrm{mm}$;

$t$ - thickness of the workpiece, mm;

$R m$ - tensile strength, MPa;

$L-$ orientation between edges of the bending matrix, $\mathrm{mm}$;

If $L=45 \mathrm{~mm}$ (7) is chosen, for small parts we get the maximum force required: $F=1.6 \cdot 500 \cdot 36 \cdot 450 / 45=288000 \mathrm{~N}$. For large parts $F=1.6 \cdot 1200 \cdot 36 \cdot 450 / 45=691200 \mathrm{~N}$. For complex parts with bending line $3000 \mathrm{~mm} F=1.6 \cdot 3000 \cdot 36 \cdot 450 / 45=1728000 \mathrm{~N}$.

No calculations are shown, if you need a 6 times more powerful press, then for small parts $\mathrm{C}=C_{0}+k \cdot \Delta F$, where $C_{0}$ is the minimum price, $k$ is the coefficient, and $\Delta F$ is the difference between the forces. Hydraulic presses with a force of $100000 \mathrm{~N} C_{0}$ are about $50000 \mathrm{EUR}$ and the price of $2000000 \mathrm{~N}$ press $C_{0}$ is about 150000 EUR [8]. The coefficient depends on the equipment and is priced at $0.53 \mathrm{EUR} / \mathrm{N}$.

For small parts, a press with a force of $300000 \mathrm{~N}$ is enough and the press price could be $C m=50000+0.53 \cdot 20000=50000+10600=60600$ EUR. For folding large parts, a $700000 \mathrm{~N}$ press for $\mathrm{Cl}=50000+0.53 \cdot 600000=50000+31800=81800$ EUR would be appropriate. The press for $C s l=50000+0.53 \cdot 190000=50000+10070=150700$ EUR would be suitable for folding complicated parts. The results show that the calculated costs for different types of parts could be significantly lower, if less presses were used, lower price of the press and equipment, the power of the electric motor also reduces the total production costs and increases the company's profit and competitiveness in the market.

For example, recalculating the machine hour cost for folding small parts, we get:

$C O S=(60600+30000) / 5+90600 / 5+90600 \cdot 0.07+7 \cdot 0.1 \cdot 0.20 \cdot 8 \cdot 1700+576=31472$ EUR.

Machine hour cost will be: $\operatorname{COSh}=31472 / 1853=16.98$ EUR per hour against 31.73 EUR per hour according to the experimental data, if the $1200000 \mathrm{~N}$ press is used.

These calculations are made on condition that the press is used for mass production. In serial production, the completion time of the work, consisting of the press shutdown, program removal, punching and matrix dismantling and tool delivery, is also assessed. Using Table 1 data it can be calculated that in the case of small parts the completion time of the work is 2.74 min., in the case of large parts the completion time of the work is $4.69 \mathrm{~min}$, in the case of complex part folding work it is $18.28 \mathrm{~min}$.

For serial production, small series are processed for up to 4 hours. We calculate how many Smol parts can be made in $4 \mathrm{~h}$, if we consider that production does not take place during production preparation, work completion and rest.

$240 \min -3.62-2.74-16.8=217.58 \mathrm{~min}$. 
$(2.85+2.38+0.32) / 10=0.555 \mathrm{~min} .217 .58 / 0.555=392$ det. An average of 49 parts can be made in one hour. To pay off, the price must be no less than $43.11 / 49=0.88$ EUR.

The small series of large parts handles $240-5.44-4.69-16.8=213.07 \mathrm{~min}$. During this time,

$214.76 /(11.57-5.45)=214.76 / 6.12=34.80$ parts can be made. 8.70 parts can be made within an hour. To pay off, the price must be no less than $43.11 / 8.70=4.955$ EUR. The minimum number of parts in a series that is economically reasonable is 9 large parts.

Small series of complex parts processing: $240-19.03-18.28-16.8=185.89 \mathrm{~min}$. During this time, $185.89 /(39.65-19.03)=185.89 / 20.62=9.02$ parts can be made. An hour can produce 2.35 parts. To pay off, the price must be no less than 43.11/2.35 $=18.34$ EUR. The minimum number of parts in a series that would be economically reasonable is 3 complex parts.

For medium and large series production, the times of preparation and completion of works are less important and can be estimated.

Calculations show that the cost of the press and equipment for folding works can be recovered by working in one shift, if they are used all the time. If the press is only used for half the working time, the cost of machine hours increases by about 1.6 times.

Using the press in two shifts, on similar terms, the cost of purchasing the press and equipment will pay off in about 2.5 years.

In cases where the number of parts in a series is below the calculated minimum, it is recommended to seek cooperation with specialised companies or to increase the price of folding works.

\section{Conclusions}

1. The existing bending techniques are best suited for small part production in small, medium and large series production.

2. The technology of bending large and complex parts does not exclude the use of the unused work time of $11 \%$ and $29 \%$ of the total time, respectively. It increases, when several folds are made that are not in the same plane, when different curve radii are to be formed and when it is necessary to manipulate a heavy part.

3. Choosing the right press force makes it possible to significantly reduce the cost of machine time.

4. Mechanization tools and robotics should be used more efficiently for complex and large part bending.

5. Full use of the press machine time makes it possible to reduce the production costs and increase the company's profit and competitiveness in the market. If the press is only used for half the working time, the cost of the machine hours increases by about 1.6 times.

\section{References}

[1] MTM Methods-Time measuring testing (2017). [online][11.02.2020] Available at: www.fcesoftware.com/images/47 MTM tests.pdf.

[2] MK noteikumi Nr.1528, no 2013.g.17.dec.

[3] Alan Rushton, Phil Croucher, Peter Baker. The handbook of logistics and distribution management. 3rd edition. London and Philadelphia. The Chartered Institute of Logistics and Transport (UK) $2006.612 \mathrm{p}$.

[4] Byrne H. Work Measurement Methods. North Carolina State University, 2014.

[5] Lawrence S. Work Measurement and Methods improvement. John Wiley\&Sons, Inc., 2000, $452 \mathrm{p}$.

[6] Handbook for metal construction technology. Herausgegeben von Armin Steinmuller. 5.Auflage. Verlag Europa-Lehrmittel.2007. 440 s. (In German)

[7] Time Study Technique REFA (2017) [online][11.02.2020] Available at: http://ww.refa.de/home.

[8] Berziņš M.Bending part production time rationing methodology: master thesis. Jelgava: Latvian University of Agricuture, 2017. 84 p.

[9] Busher Municipal AG. [online][11.02.2020] Available at: www.buchermunicipal.com/en. 